\title{
American Academy of Sleep Medicine Guidelines, 2018
}

\author{
Anand Singh ${ }^{1}$, Harsh Meshram², Milk Srikanth ${ }^{3}$
}

\begin{abstract}
Quality of care should be improved by following the clinical guidelines across the world. Guidelines have potential benefits not only for patients but also for clinicians and healthcare system in formulating the health schemes. Clinicians with sound knowledge need to identify the specific barriers, beyond knowledge, that stand in the way of behavioral change. Recent American Academy of Sleep Medicine (AASM) guidelines 2018 have come up with new recommendations such as split night polysomnography (PSG), repeat PSG in strongly suspected individuals with initial negative PSG and more to improve the specificity of diagnosis.

Keywords: AASM guidelines 2018, Clinical guidelines, Quality of care.

International Journal of Head and Neck Surgery (2019): 10.5005/jp-journals-10001-1379
\end{abstract}

\section{Diagnostic Testing for Obstructive Sleep Apnea ${ }^{1}$}

- Obstructive sleep apnea (OSA) can only be diagnosed by polysomnography (PSG). Questionnaires are not sufficient to diagnose OSA

- PSG should be done in place of home-based sleep tests (HBST) for patients with cardiovascular diseases/respiratory disease/ neuromuscular conditions/stroke, which may bring about night time desaturation

- If HBST do not detect OSA in individuals with formidable risk of OSA, PSG must be done subsequently $(\mathrm{N})$

- Split night PSG may be used if high probability of OSA present and the subsequent criteria are satisfied ( $\mathrm{AHI}>40$ for minimum 2 hours, CPAP titration is done for minimum of 3 hours, PSG demonstrates that CPAP has abolished OSA) (N)

- If first PSG fails to detect OSA, subsequently PSG can be repeated if OSA is suspected (N).

\section{INDICATIONS FOR PSG ${ }^{1}$}

- Patients with cardiac failure (systolic/diastolic) with nocturnal symptoms or insignificant improvement despite optimal medical therapy should undergo PSG $(\mathrm{N})$

- Patient with coronary artery disease/cerebrovascular disease/ arrhythmias should be evaluated with PSG if symptoms/signs of OSA are present $(\mathrm{N})$

- Titration of pressure setting on positive airway pressure (PAP) device for patient

- Pre-procedural testing prior to operative treatment for OSA/ upper airway resistance syndrome (N).

\section{Follow-up Testing in Special Circumstances}

- In patients who have good clinical improvement after use of oromandibular devices in cases of moderate/severe OSA

- After operative intervention in patients with moderate/severe OSA

- In patients with SRDB who become symptomatically worse following initial improvement after operative intervention ${ }^{1}$ Department of Respiratory Medicine, London North West University
Healthcare NHS Trust-Ealing Hospital, London, UK
${ }^{2,3}$ Department of Chest Medicine, Lilavati Hospital and Research
Center, Mumbai, Maharashtra, India

Corresponding Author: Anand Singh, Department of Respiratory Medicine, London North West University Healthcare NHS Trust-Ealing Hospital, London, UK, Phone: +44 2089675000, e-mail: anand69_ace@ hotmail.com

How to cite this article: Singh A, Meshram H, Srikanth M. American Academy of Sleep Medicine Guidelines, 2018. Int J Head Neck Surg 2019;10(4):102-103.

Source of support: Nil

Conflict of interest: None

- In individuals who have substantial alteration in body weight (10\% gain or loss) to regulate CPAP pressure settings

- If initial improvement with CPAP use is followed by re-emergence of symptoms (testing is done to look for concomitant second sleep pathology such as central sleep apnoea/narcolepsy).

\section{Actigraphy ${ }^{2}$}

This provides a means of assessing sleep architecture and sleep duration in individuals. It can accurately diagnose circadian sleep disorders such as shift work disorder and delayed sleep phase syndrome. It is not reliable to diagnose OSA. In pediatric population/ geriatric population when PSG is unavailable/technically difficult, actigraphy can be an useful adjunct.

\section{Multiple Sleep Latency Test/Maintenance of Wake Fullness Test ${ }^{3}$}

These tests are indicated for individuals suspected to have narcolepsy/insomnia/circadian sleep disorders. These tests do not correlate with accident risk in real world and that clinical wisdom is more consistent to judge this $(\mathrm{N})$.

\section{Treatment of OSA OS-10 $^{4}$}

\section{PAP Therapy}

\section{CPAP}

This is the first-line treatment for moderate and severe OSA. It is an option for mild OSA (apnoea hypopnoea index < 15); however,

(-) The Author(s). 2019 Open Access This article is distributed under the terms of the Creative Commons Attribution 4.0International License (https://creativecommons. org/licenses/by-nc/4.0/), which permits unrestricted use, distribution, and non-commercial reproduction in any medium, provided you give appropriate credit to the original author(s) and the source, provide a link to the Creative Commons license, and indicate if changes were made. The Creative Commons Public Domain Dedication waiver (http://creativecommons.org/publicdomain/zero/1.0/) applies to the data made available in this article, unless otherwise stated. 
studies done have shown inconsistent results. The patients started on CPAP should be followed up in the clinic to monitor compliance and address issues related to CPAP such as humidification, mask/ machine-related trouble. CPAP usage can be objectively measured as new devices have memory chips that store data.

\section{BPAP}

Bi-level positive airway pressure devices are used to treat obesity hypoventilation syndrome and OSA associated with restrictive lung disease such as kyphoscoliosis.

\section{Auto-CPAP (APAP)}

APAP should not be used to diagnose OSA in titration studies or split night studies. Patients who have cardiovascular comorbidities contributing to desaturation should not use APAP. Individuals on fixed CPAP therapy settings as determined by APAP should be under clinical followup and should be considered for repeat polysomnography if symptoms do not resolve.

APAP devices can be used in self-adjusting mode for unsupervised treatment of OSA patients without cardiovascular/ pulmonary comorbidities. APAP devices can be used to determine optimal pressure required for OSA patients without comorbidities; however, manual CPAP devices during polysomnography remain the gold standard.

\section{Oromandibular Devices}

They are the next best treatment option after CPAP. These devices should be prepared by qualified dentists. These patients should be followed up by sleep physicians to corroborate improvement clinically and on sleep study.

\section{Medical Therapy of OSA}

Weight loss via diet and exercise is essential for treatment of OSA and must be supplemented with definite treatment for OSA. (N) Topical nasal corticosteroids can reduce the $\mathrm{AHI}$ in individuals with concomitant rhinitis and OSA. It is now a useful adjunct therapy in OSA. ${ }^{4-10}$

\section{Treatment for Central Sleep Apnea (CSA)}

\section{CSA secondary to CHF (congestive heart failure)}

- CPAP is initially used. Nocturnal oxygen therapy is a helpful adjunct in this setting

- (N)Adaptive Servo-Ventilation (ASV) is as effective as CPAP therapy and should be used if ejection fraction is $>45 \%$. (Evidence shows increased mortality in cases of low ejection fraction)

- BPAP therapy in a spontaneous timed (ST) mode can be used when there is suboptimal response to CPAP, nocturnal oxygen, and ASV

- Acetazolamide and theophylline have very limited evidence to support their use. They may be considered as adjunctive therapy $(\mathrm{N})$.

\section{Primary CSA}

- The data supporting PAP therapy (CPAP, BPAP-ST, and ASV) are inadequate; however, it is unlikely to cause any harm and can be used $(\mathrm{N})$

- Acetazolamide may be used, but there is minimal evidence to demonstrate its efficacy

- If a patient does not have risk factors for respiratory depression zolpidem, triazolam may be used however vigilant followup is essential $(\mathrm{N})$.

CSAS related to end-stage renal disease $(\mathrm{N})$

- Options available are CPAP, supplemental oxygen, bicarbonate buffer use during dialysis, and nocturnal dialysis. ${ }^{4-10}$

\section{References}

1. Kapur VK, Auckley DH, Chowdhuri S, et al. Clinical practice guideline for diagnostic testing for adult obstructive sleep apnoea: an American Academy of Sleep Medicine clinical practice guideline. J Clin Sleep Med 2017;13(3):479-504. DOI: 10.5664/jcsm.6506.

2. Morgenthaler T, Alessi C, Friedman L, et al. Practice Parameters for the Use of Actigraphy in the Assessment of Sleep and Sleep Disorders: An Update for 2007. SLEEP 2007;30(4):519-529. DOI: 10.1093/sleep/30.4.519.

3. Littner MR, Kushida C, Wise M, et al. Standards of Practice Committee of the American Academy of Sleep Medicine. Practice parameters for clinical use of the multiple sleep latency test and the maintenance of wakefulness test. SLEEP 2005;28(1):113-121. DOI: 10.1093/sleep/ 28.1.113.

4. Kushida CA, Littner MR, Hirshkowitz M, et al. Practice parameters for the use of continuous and bilevel positive airway pressure devices to treat adult patients with sleep-related breathing disorders. SLEEP 2006;29(3):375-380. DOI: 10.1093/sleep/29.3.375.

5. Morgenthaler TI, Aurora RN, Brown T, et al. Standards of Practice Committee of the AASM. Practice parameters for the use of autotitrating continuous positive airway pressure devices for titrating pressures and treating adult patients with obstructive sleep apnea syndrome: An update for 2007. SLEEP 2008;31(1):141-147. DOI: 10.1093/ sleep/31.1.141.

6. Kushida CA, Chediak A, Berry RB, et al. Positive Airway Pressure Titration Task Force of the American Academy of Sleep Medicine. Clinical guidelines for the manual titration of positive airway pressure in patients with obstructive sleep apnea. J Clin Sleep Med 2008;4(2):157-171.

7. Ramar K, Dort LC, Katz SG, et al. Clinical practice guideline for the treatment of obstructive sleep apnea and snoring with oral appliance therapy: an update for 2015. J Clin Sleep Med 2015;11(7):773-827. DOI: $10.15331 / \mathrm{jdsm} .4868$.

8. Morgenthaler TI, Kapen S, Lee-Chiong T, et al. Practice parameters for the medical therapy of obstructive sleep apnea. SLEEP 2006;29(8):1031-1035. DOI: 10.1093/sleep/29.8.1031.

9. Aurora RN, Bista SR, Casey KR, et al. Updated adaptive servo-ventilation recommendations for the 2012 AASM guideline: "The Treatment of Central Sleep Apnea Syndromes in Adults: Practice Parameters with an Evidence-Based Literature Review and Meta-Analyses". J Clin Sleep Med 2016;12(5):757-761. DOI: 10.5664/jcsm.5812.

10. https://aasm.org/clinical-resources/practice-standards/practiceguidelines/. 